\title{
Time Domain SAR Raw Data Simulation of Distributed Targets
}

\author{
Ozan Dogan ${ }^{1,2}$ and Mesut Kartal ${ }^{2}$ \\ ${ }^{1}$ Informatics Institute, Istanbul Technical University, Maslak, 34469 Istanbul, Turkey \\ ${ }^{2}$ Electrical and Electronics Faculty, Istanbul Technical University, Maslak, 34469 Istanbul, Turkey
}

Correspondence should be addressed to Ozan Dogan, ozan.dogan@be.itu.edu.tr

Received 3 May 2010; Revised 1 September 2010; Accepted 20 September 2010

Academic Editor: M. Greco

Copyright ( 2010 O. Dogan and M. Kartal. This is an open access article distributed under the Creative Commons Attribution License, which permits unrestricted use, distribution, and reproduction in any medium, provided the original work is properly cited.

In this paper, a time domain stripmap mode Synthetic Aperture Radar (SAR) raw data simulation including both the terrain and the targets is proposed. The simulator generates SAR raw data of a scene, involving both single and double reflections in a computationally efficient manner. The inputs of the simulator are the digital elevation model of the terrain, the 3D target model, and the parameters of the SAR system. The simulator extracts a geometrically accurate reflectivity map and generates the SAR raw data in time domain. The disadvantage of time domain method is justified to be tolerable by presenting experiments on modularity performance of the simulator. Also a novel method to decrease the time domain computational complexity of the SAR raw data generation is proposed. Our method has showed very promising results in representing the scattering characteristics, the raw data, and the time domain simulation flexibility.

\section{Introduction}

SAR is a powerful remote sensing technique that enables monitoring of surface by using the surface backscattering characteristics obtained under illumination of microwaves. SAR simulators are used in measurement of performance, design of new algorithms, hardware, and architectures, for mission planning and sensor organization, and developing image exploitation techniques. Based on those application areas, the SAR data simulators can be classified into two groups [1]: the image-oriented simulators [2-5] for the applications like mission planning, automatic target recognition, or georegistration of SAR images and signal-oriented simulators [6-9] for algorithm design. As a signal-oriented simulator, the main concern of the work in this paper is to generate the SAR raw data of the extended scenes and the targets.

There are three main expectations that a SAR simulator shall meet: (1) accuracy, (2) modularity, and (3) computational efficiency. Currently, to the best of our knowledge, there isn't any SAR simulator which is capable of satisfying all three of these objectives satisfactorily. One of the accuracy parameter of a SAR simulator can be a wide coverage of the scattering concepts for the reflection computation.
An accurate reflection computation is expected to consider the layover, foreshortening, and the shadow effects to be geometrically accurate. Moreover, it is also expected that the multiscattering, polarization, and speckle effects to be simulated physically accurate $[2,10,11]$. Frequently, the terrains are modelled statistically from the empirical values [12]. However, the target shall be considered as another case: computing single scattering is far from adequate; double scattering cases (even higher-order multibounce cases) shall also be considered [11]. Of course, computation of double and higher-order scatterings comes with considerable extracomputational load.

Observing the effects of different scenarios for the sensor and the scene configuration is the major outcome of a SAR simulator. Therefore, SAR simulators are desired to be modular, that is, flexible for different waveform trials, platform deviations, and different target shapes and orientations. While the frequency domain simulators are efficient, it is considerably more difficult to generate system transfer functions that contain the many degrees of freedom in general imaging scenarios [7-9, 13]. Another critical weakness of these methods is that they simulate the raw data by utilizing the inverse concepts of the SAR image formation algorithms like Stolt interpolation of Omega-K 
or chirp scaling. It is the fact that, evaluating an algorithm using data simulated by inverse of the algorithm is principally inadequate. Besides, time domain simulators provide a functional separation of the simulator architecture in order to represent different waveforms (like LFM, coded, etc.) and deviations in sensor trajectory.

There is, of course, a tradeoff between the accuracy, modularity, and the computational efficiency. The more the accuracy increases, the more the computational time is required, rendering the simulator unusable in time-critical applications. In applications like generation of sample data for the automatic target recognition systems, the accuracy of the reflectivity computation is very important. However, in applications like raw data processing evaluation, one seeks computational efficiency rather than accuracy of the reflectivity computation.

In this paper, we utilized the well-known shooting and bouncing ray approach [10] to compute the reflectivity of the terrain and the target. For the sake of modularity, there is no limitation about the orientation of the target. Both single and double scattering cases and the geometrical features like the layover, foreshortening, and the shadowing effects are taken into consideration. In order to increase the computational efficiency, the reflectivity estimation procedure neglects the material type of the targets and the multiscattering (more than two) cases, which makes the simulator geometrically accurate however radiometrically inaccurate. The radiometric correction is seen as a postprocessing procedure after image formation, so this effect can be ignored from a signal processing point of view. Also as the geometrical optics (GOs) principles are utilized, the simulator is considered physically accurate enough for SAR interpretation and SAR signal processing assessment too. The GO approach is inspired by the reflection of light and as a result known to be a good match for high frequencies like X band, however the lower the operating frequency, other scattering mechanism shall be taken into consideration [12].

We have built a stripmap mode SAR simulator. Given the digital elevation model (DEM) of the scene, 3D model of the target, and the SAR System parameters as input, the simulator separates the model into planar surfaces, known as facets, and obtains the surface reflectivity by estimating the Radar Cross Section (RCS) and the shadow effect for each facet. The SAR raw data is obtained by extracting the contributions of each facet to the SAR range bins. Besides, a novel time domain SAR raw data computation procedure is also proposed in this paper.

The main goal of our simulator is to evaluate different SAR image formation algorithms like Range Doppler, Chirp Scaling, and Omega-K. Unlike in [7, 9], Stolt interpolation or chirp scaling methods are not allowed for us to simulate raw data to evaluate the algorithms that utilize these methods. That is why, a time domain raw data simulation is proposed although the computational complexity heavily increases. However, this performance is also increased by proposing a novel method. Another reason of time-domain choice is the modularity advantage of these simulators. We also assessed the flexibility of our simulator in handling different scenarios like moving targets and antenna angular deviations and

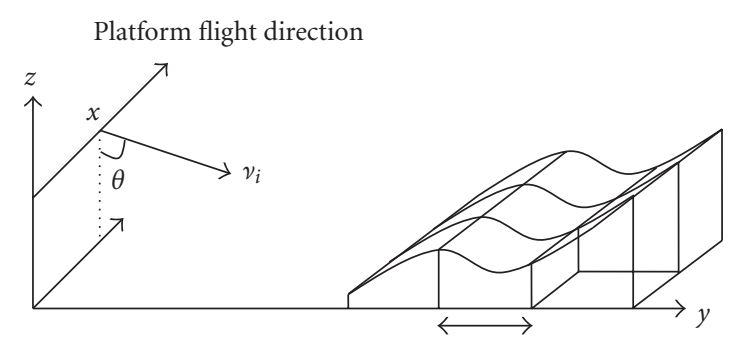

Figure 1: Geometry of flight path and the terrain.

discussed the potentialities of the proposed work to cover the platform trajectory deviations and different waveform cases. Let us note that there is no efficient SAR raw data simulator that can fulfill such different scenario requirements.

The paper is organized as follows. The reflection model is presented in Section 2. In Section 3, the raw data simulation is explained. The last two sections are the results and the conclusion.

\section{Reflectivity Model}

A model for the computation of the random-shaped target backscattering is proposed. The $3 \mathrm{D}$ inputs of the simulator are the Digital Elevation Model (DEM) for modelling the terrain and the target model. The targets are assumed to be standing on the terrain. Given the size of the target, the simulator generates the facets. Facets are known to be adequate to model the $3 \mathrm{D}$ terrain and the target [11]. For the sake of simplicity, each facet is employed as rough surface and assumed to be a scattering center, and the back scattering from each facet is computed under the Geometrical Optics (GOs) approximation in order to compute large scenes efficiently. In the double scattering case, the surface attenuation coefficient of each facet is considered. The ratio of the height deviation to the resolution of the sensor is taken into account to determine the number of double paths. For each path, the incidence angle, the scattered ray and the new incidence angle from the target to the ground or target to target (and vice versa), are computed via ray tracing. The range of this trip and the contribution to the reflectivity map is computed. The well-known narrow beam approximation is utilized to compute backscattering [12].

2.1. Single Scattering Case. The geometry of the terrain scattering is presented in Figure 1. The sensor is moving along x direction. The vector $\vec{v}_{i}$ is the propagation direction of the electromagnetic waves:

$$
\vec{v}_{i}=\sin (\theta) \vec{y}-\cos (\theta) \vec{z}
$$

where $\theta$ is the incidence angle, $\vec{y}$ and $\vec{z}$ are the unit vectors.

In the single scattering case, all facets, except the ones in the shadow, will contribute to the radar backscattering. The reflectivity of the surface and the target are modelled as the cosine of the angle between the incidence vector and the normal vector. This model is also known as the Lambertian approach and is used in many works like $[3,4,7]$. 


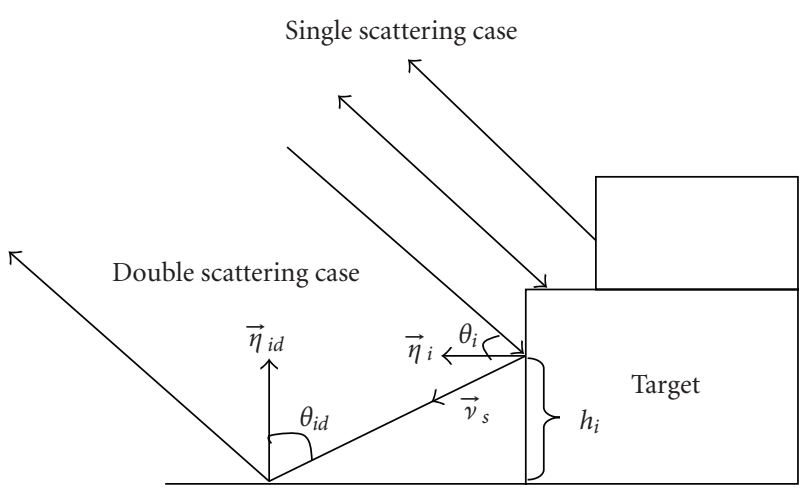

FIgURE 2: Target cross-section in $y-z$ plane.

The terrain is a two-dimensional function of height $z=$ $f(x, y)$, where $x$ is the azimuth direction and $y$ is the ground range. The surface normal is simply the vector multiplication of the two vectors that are related with the gradients in $x$ and $y$ directions and the model resolution as follows:

$$
\vec{n}_{i}=\frac{(d f(x, y) / d x) \vec{x}+(d f(x, y) / d y) \vec{y}-\delta_{m} \vec{z}}{\sqrt{(d f(x, y) / d x)^{2}+(d f(x, y) / d y)^{2}+\delta_{m}^{2}}}
$$

where $\delta_{m}$ is the digital elevation model spatial resolution assumed to be equal in $x$ and $y$ direction, the index $i$ is the facet index, and $\vec{x}$ is the unit vector.

Until now, the basic terrain scattering mechanisms are discussed. To model target reflectivity, one shall consider both the edges, vertical surfaces, and double scattering from the vertical surfaces of targets. The geometry of the backscattering phenomena for a target is presented in Figures 2 and 3 for different projections. A 2D cross-section of the scenario is shown in Figure 2. Single- and double-scattering cases are also shown in that figure.

2.2. Double Scattering Case. The target backscattering from the top and the edges of the target is named single-reflection contributions and has been widely studied in the literature. However, knowledge of the interactions between the vertical feature of the targets and the terrain are not so mature. In this case, one shall also take double-scattering cases into account. In [14], a very impressive work on SAR raw data simulation of urban area, the importance of the multi-scattering case was emphasized and an analytical expression for both GO and Physical Optics (POs) approaches were given for a parallel-epiped shaped target. However in contrast with [11], in this simulator the orientation dependency of the double reflection is neglected. When the target vertical features are parallel to the azimuth direction, the double scattering contribution is expected to generate a line while when the target vertical features are oriented the contribution must be diffused. In [11], this smear effect is neglected. Nevertheless in this work the shape of the target is assumed to be geometrically known exactly and the shape is restricted to a rectangular prism. The 3D models of the targets are mostly not canonical shapes that the sizes are exactly known.

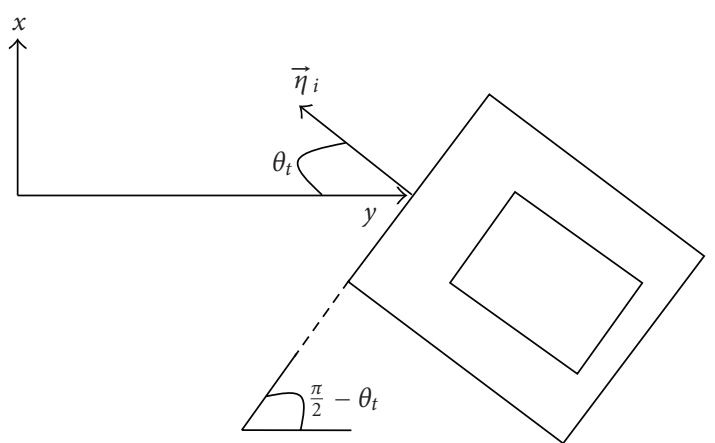

Figure 3: Target cross-section in $x-y$ plane.

That is why in our paper, we proposed a procedure to separate the walls into facets and each facet double scattering contribution is computed individually. This capability makes our approach expandable to cover the more complicated scattering mechanisms of nonideally parallel-epiped shaped targets like it is shown in Figure 2.

In this paper, we formulized the double-scattering case, by modelling the interactions between the target and the ground. The number of facets is determined by a criterion that is inspired from the Rayleigh criteria. The ratio of height deviation through the target to the radar resolution is computed to determine the number of facets. If this value is more than a predefined sensitivity factor, the surface is considered as a vertical surface and the double scattering case is taken into account. The number of facets is computed via $m=\lceil\Delta h / \Delta r\rceil$ where $\Delta h$ is the height deviation in $y$ direction, $\Delta r$ is the range resolution, $s$ is the sensitivity factor that is related with the facet size in the vertical surfaces of the target, and \lceil\rceil is the ceiling operator in order to guarantee $\mathrm{m}$ to be an integer. For each facet, the surface normal, the scattering vector, the point at which the ray touches the ground, the backscattering vector, and the full range path are computed as follows.

The normal of the vertical surfaces of the target is assumed to be a vector in the $x-y$ plane. The surface normal, as it is shown in Figure 3, is related with the orientation of the target. The normal vector is formulated as

$$
\vec{n}_{i}=\sin \left(\theta_{t}\right) \vec{x}-\cos \left(\theta_{t}\right) \vec{y}
$$

Then utilizing the Snell's Law, the direction of propagation of the specularly reflected signal, $\vec{v}_{s}$, is simply

$$
\vec{v}_{s}=\sin (\theta) \sin \left(2 \theta_{t}\right) \vec{x}+\sin (\theta) \cos \left(2 \theta_{t}\right) \vec{y}-\cos (\theta) \vec{z} .
$$

The next step is to compute the point that the ray intersects the ground. In the $z$ direction, the time required for the ray to reach the ground is $t_{d}=\left(h_{i} / \cos (\theta)\right)(1 / c)$ where $c$ is the speed of light and $h_{i}$ is the height of the $i$ th facet. Then, as the time for the pulse to reach the ground is known, point 
that the ray intersects the ground can be easily formulated by computing the ray path distance in $x$ and $y$ directions:

$$
\left(x_{t d}, y_{t d}\right)=\left(x_{t}, y_{t}\right)-\left(\sin (\theta) \sin \left(2 \theta_{t}\right), \sin (\theta) \cos \left(2 \theta_{t}\right)\right) c t_{d},
$$

where the index $d$ indicates the double scattering case. Since the surface normal and the incidence vector are both known, the amplitude of backscattering that is the cosine of the angle between these two vectors can be computed simply by vector multiplication.

2.3. Construction of Reflectivity Matrix. The final reflection coefficient is defined as both the amplitude and the phase term

$$
\gamma(x, r)=|\gamma(x, r)| \exp (-j \zeta(x, r))
$$

where $|$.$| is the amplitude operator and \zeta$ is the phase term:

$$
\zeta(x, r)=\frac{4 \pi r}{\lambda}-\varphi(x, r),
$$

where $\varphi(x, r)$ is a random, zero mean Gaussian process with standard deviation of one and $r$ is the range between the target and sensor. This equation explicitly connected with the phase deviation regarding the surface roughness. Modelling roughness is important especially in the case of imaging the targets situated over or between rough surfaces. The rougher the surface becomes, more deviation is induced between the phases of the scattering centers.

Next step is to calculate the range that the radar measures the computed reflection. The minimum range between the ideal sensor flight path and the facet edge is found by

$$
R_{i}=\sqrt{\left(h_{p}-h_{t}\right)^{2}+\left(y_{0}+i \Delta r\right)^{2}}
$$

where $h_{p}$ and $h_{t}$ are the sensor and the facet altitude, respectively, and $y_{0}$ is the minimum distance in ground. For the double-scattering case, the computed backscattering is added to the reflection coefficient matrix in the range that is the half of total ray path. Also our approach for double-scattering estimation assumes that vertical features are smooth surfaces.

\section{Raw Data Model}

3.1. Raw Data Computation Method. The SAR raw data can be modelled for stripmap mode as follows [7]:

$$
h\left(x^{\prime}, r^{\prime}\right) \quad=\iint \gamma(x, r) g\left(x^{\prime}-x, r^{\prime}-r, r\right) d x d r,
$$

where

$$
\begin{aligned}
g\left(x^{\prime}-x, r^{\prime}-r, r\right) \\
=\exp \left(-j \frac{4 \pi}{\lambda} r\right) \exp \left(j \pi \frac{2 \alpha}{c^{2}}\left(r^{\prime}-r\right)^{2}\right) w^{2}\left(\frac{x^{\prime}-x}{X}\right) \\
\quad \times \operatorname{rect}\left(\frac{r^{\prime}-r}{c \tau / 2}\right),
\end{aligned}
$$

where $x^{\prime}$ and $r^{\prime}$ are the spatial domain parameters in azimuth and in range, respectively, $\gamma(x, r)$ is the scene reflectivity map, the exponential terms are the shifted linear frequency modulated signals, while the $w^{2}$ term represents the twoway antenna azimuth pattern, $X$ term is the antenna azimuth footprint, the rectangular symbol rect() represents the range window of the pulse, $x$ is the azimuth position of the target, $r$ is the distance between the target and the flight path, the range direction in cylindrical coordinates, $r=$ $\sqrt{r_{0}^{2}+\left(x-x^{\prime}\right)^{2}}$, where $r_{0}$ is the minimum distance between the sensor and the target, $\lambda$ is the wavelength, $\alpha$ is the transmitted signal chirp rate, $\tau$ is the pulse width and $c$ is the speed of light.

We have noticed that the most time-consuming part of the raw data simulation procedure is to compute the exponential phase term for each target, for each azimuth and range position. Our idea is simply to generate the transmitted signal in a high sampling rate sufficient to generate a reasonably low azimuth phase error before the raw data generation loops. Once the highly sampled data is generated, according to the range of the target, the data is decimated and is summed up to the raw data. So the algorithm gets rid of computation of the exponential phase terms from pulse to pulse and a considerable efficiency is achieved.

The sampled range spatial domain parameter of a target can be written as follows:

$$
r^{\prime}=r+k \frac{c}{2 f_{s}}
$$

where $r$ is the range to the target, $f_{s}$ is the sampling rate and $k$ is the integer sampling index where according to the rectangular function, $k\left(c / 2 f_{s}\right) \in((-c \tau / 4),(c \tau / 4))$. In this equation it is seen that if the distance between two targets is $k\left(c / 2 f_{s}\right)$, these two targets will have the same phase shifts, but different range shifts. So this equation can be written as a phase shift and range bin shift terms:

$$
r^{\prime}=r_{\text {ref }}+n \frac{c}{2 f_{s}}+m \frac{c}{2 f_{\text {ups }}}+\varepsilon_{r}
$$

where $r_{\text {ref }}$ is the reference range, (for example range to the scene center), $n$ is the range bin of the target, $m$ is named as the phase shift bin, $f_{s}$ is the sampling rate, $f_{\text {ups }}$ is named as upsampling rate, a higher sampling rate comparing with $f_{s}$, and $\varepsilon_{r}$ is the error. Here $n$ and $m$ are integers. The azimuth signal in broad side geometry can be evaluated by just the quadratic terms. So the signal shape is a linear frequency modulated signal, named as chirp in radar terminology. In (12), if the error, notated by $\varepsilon_{r}$, can be low sufficient to form a chirp signal in azimuth direction, then a considerable computational effort is meant to be reduced for raw data simulation. Let us note that $\varepsilon_{r}<\left(c / 4 f_{\text {ups }}\right)$ as $m$ is a rounded value.

The SAR raw data is a range bin shifted and also phaseshifted version of the transmitted signal. The range bin shift amount is given by the rectfunction in (9). The index of the corresponding range bin is $n$ in (12). As it is mentioned before, if the distance between two targets is at an amount of 
TABLE 1: Simulation parameters.

\begin{tabular}{lc}
\hline Parameter Name & Value \\
\hline PRF, Hz & 300 \\
Bandwidth, MHz & 50 \\
Range, km & 20 \\
Wavelength, m & 0.03 \\
Platform Velocity, m/sn & 60 \\
Sampling Frequency, $\mathrm{MHz}$ & 60 \\
\hline
\end{tabular}

the sampling interval, there will be no need to regenerate the sampled raw data; just a shift in range will handle the phase deviation. However if the distance is below the sampling interval, as it is mostly the case, a more detailed analysis must be done. Because, this shift is the main reason of the azimuth signal to be a chirp signal. In this paper we proposed that, an up sampling rate, $f_{\text {ups }}$, can be set in a manner to generate an allowable error.

3.2. Method Verification. As it is seen in (12) the maximum value of error for each pulse is the half of the upsampling interval:

$$
\varepsilon_{r}(\max )=\frac{c}{4 f_{\text {ups }}} .
$$

In [15], the maximum allowable quadratic phase error is determined as $\pi / 2$ radian for the azimuth aperture. So one can claim a total maximum allowable phase error criterion to be

$$
N \frac{4 \pi}{\lambda} \varepsilon_{r}<\frac{\pi}{2}
$$

where, $N$ is the number of pulses. If the maximum value of the error is assumed to be produced for each pulse, the limitation for the upsampling rate is

$$
f_{\text {ups }}>\frac{2 c N}{\lambda}=\frac{2 c r_{0} \theta_{a} f_{a}}{\lambda v},
$$

where $\theta_{a}$ is the antenna beamwidth, $f_{a}$ is the pulse repetition frequency, and $v$ is the platform velocity. So if the upsampling rate is selected sufficiently high, the $\pi / 2$ radian phase error can be achieved. As the phase error is distributed nonlinearly, there is no analytical exact solution. However, the phase error is distributed between zero and the maximum value, and the mean value can be taken intuitively as the half of the maximum value.

The proposed method has impact on both terms in (9). The rate between the sampling rate with the up sampling rate is sufficiently high and results in a low range shift error compared with the range bin. To evaluate these effects, assume the following configuration according to the parameters given in Table 1.

In this case, the maximum spatial error is approximately $0.35 \mu \mathrm{m}$. Comparing this value with the range sampling interval, which is $2.5 \mathrm{~m}$ for this case, shows that the impact of the approach to the terms other than the azimuth term in (9) can be neglected. So the main discussion will be on azimuth phase term.

The ratio between the upsampling rate and sampling rate is notated as oversampling ratio (OSR). The simulation results show that when OSR is lower than the limitation, the azimuth signal is still a chirp signal. So to evaluate the level of "chirpiness", we proposed to utilize the matched filter to find the correlation between the exact chirp and the approximate chirp. Here the correlation procedure is also known as the pulse compression in radar conventions. It is known that the best achievable peak to side lobe ratio (PSLR) is $-13.3 \mathrm{~dB}$ after compression [16]. In our work it is observed that when OSR is sufficiently high, (at least half the upsampling ratio that satisfies (15)) the expected PSLR is achieved. So the intuitive approach is verified empirically. Above this OSR also do not produce more PSLR as it is expected. So our criterion is valid, because the proposed method converges to the best achievable PSLR that can be obtained by the exact time domain simulation.

The fundamental idea of our approach is to generate the transmitted signal in a very high sampling ratio and store the decimated version before raw data generation. Raw data is generated by shifting the appropriate decimated data and finally demodulated to baseband.

\section{Numerical Results}

The flowchart of the simulator is presented in Figure 4. The digital elevation model, target model, and the sensor parameters are the inputs of the simulator. The flow includes the whole process from reflectivity map generation to raw SAR data generation.

The flow of the simulator is as follows. The simulator firstly computes the shadow regions with a ray tracing method. If the point of interest is not in shadow, the single- and, if available, the double-scattering is computed with the same ray tracing procedure. Then the ranges to each target are computed, and the corresponding range and phase-shifted transmitted signal is summed up to the related range bin. The SAR raw data is extracted after a heterodyne operation. At last, in order to observe the final SAR image, image formation is done by the range and azimuth compression, respectively.

In order to show some of the potentialities of the simulator, in the next subsections, we present some simulation experiments. The experiments to show the capabilities of the simulator in the interpretation of SAR images are explained. Also a validation procedure is discussed. The modularity advantage of a time domain SAR simulator that handles two different scenarios is given: angular deviation of antenna and moving targets. Last subsection is the comparison of our proposed work with the other SAR simulators.

4.1. Simulator Validation. Validation is done by applying the procedure of [14] to the proposed simulator. In the first set of experiments, we have simulated the SAR raw signal from a scene composed of a target with height $73.5 \mathrm{~m}$, azimuth dimension of $40 \mathrm{~m}$. The simulator parameters are presented 


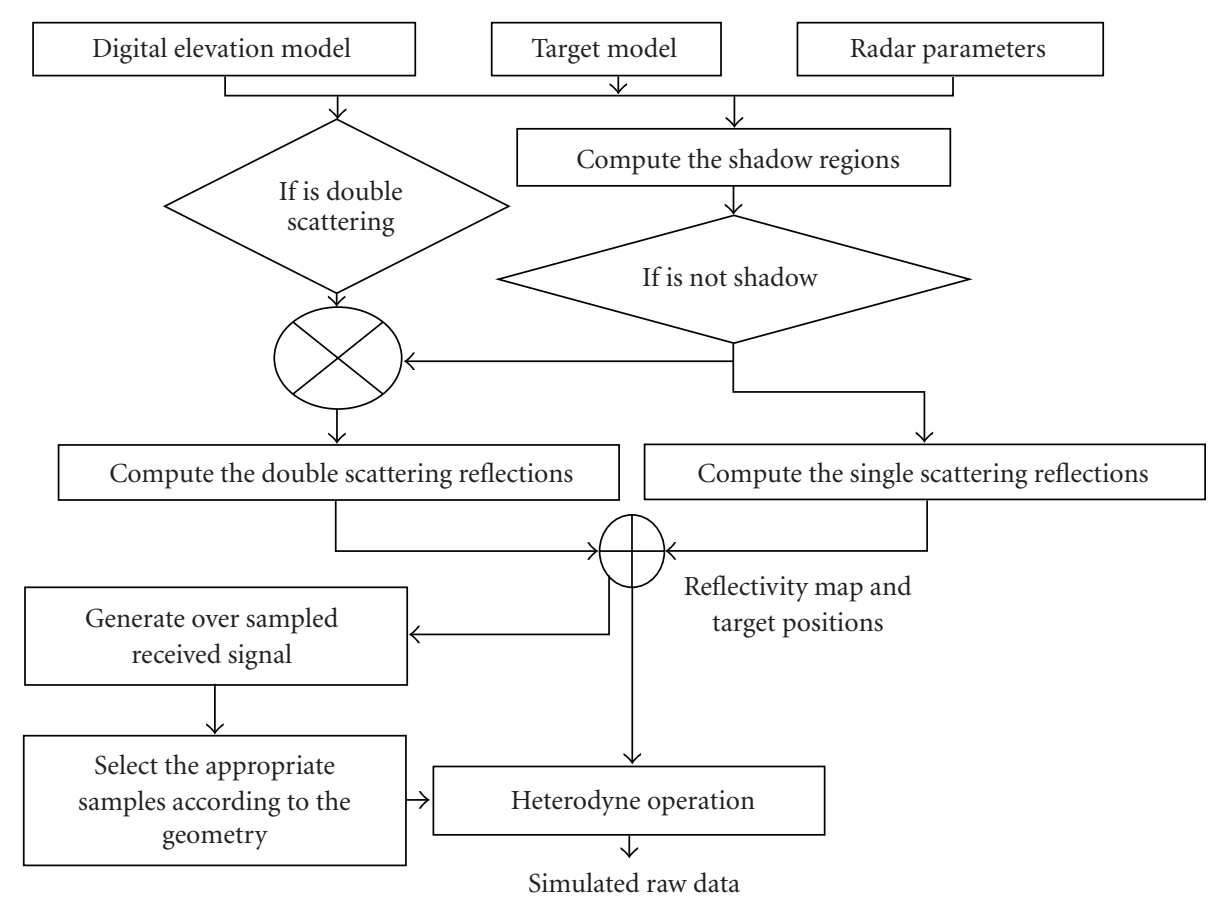

FIGURE 4: The flow of the SAR raw data simulation and image formation.

in Table 2. In Figure 5, the 3D model of the target, the radar position, the equal-range curves and the corresponding radar fast time is shown. We note that, as it is presented in the figure, the shape of the roof is not necessarily flat which is the case for [14]. Before the time $t_{1}$, the backscattering to the radar is sourced by the background. In the $\left(t_{1}, t_{2}\right)$ interval, the vertical features of the target causes the backscattering. $t_{2}$ is a critical time in two aspects. firstly the roof effect starts in this time. Secondly, peculiar to this geometry that the walls are parallel to the azimuth direction, the double-scattering contributions run optical paths of the same length, so they are grouped in the time instant whatever the illumination angle and the building height are. $\left(t_{3}, t_{4}\right)$ is the interval that the incidence angle is higher comparing with the previous interval and as a result the power of backscattering decreases. This interval is also candidate to the start of the shadow regarding the aspect angle. $\left(t_{4}, t_{5}\right)$ is the time that the shadow is guaranteed to start due to the slant geometry of SAR. From $t_{5}$ on, the only contribution to the reflectivity function will be given by the ground scattering. We emphasize that as the aspect angle, $\theta$, decreases all the intervals also decrease. The reduction in $\left(t_{1}, t_{3}\right)$ interval gives rise to a foreshortening phenomenon and when the two are displaced to the layover effect while the reduction in $\left(t_{3}, t_{5}\right)$ interval shortens the shadowed area.

Note that the sequencing of these times may change according to the incidence angle, target shape, and size. For instance, $t_{1}$ may be less than $t_{3}$ resulting in a layover effect or $t_{2}$ may be greater than $t_{3}$, meaning a double-bounce contribution in the shadowed area.

In Figure 6, the final simulated SAR image obtained by a range of $20 \mathrm{~km}$, corresponding to a $75.5^{\circ}$ aspect
TABLE 2: Simulator parameters.

\begin{tabular}{lc}
\hline Parameter Name & Value \\
\hline Frequency, GHz & 10 \\
PRF, Hz & 500 \\
Pulse Width, $\mu$ s & 1 \\
Sensor Height, $\mathrm{m}$ & 5000 \\
Model Resolution, m & 1 \\
SAR Resolution, $\mathrm{m}$ & 1 \\
\hline
\end{tabular}

angle is shown. From left to right, in range direction, we can recognize the layover area, then the brightest doublereflection line, the building roof contribution, and the shadow. The aspect angle is higher than the corresponding roof angle which is $71.47^{\circ}$. So, after the time $t_{3}$, instead of $t_{4}$, the shadow occurs because the downslope part of the roof cannot be illuminated.

The simulated SAR image for an aspect angle of $51.3^{0}$ (corresponding to $8 \mathrm{~km}$ range) is presented in Figure 8 . Comparing this image with the previous one, we observe that the down slope part of the roof is not shadowed as an expected result. As the aspect angle decreases, the time intervals decrease and the upslope part of the roof and also the shadow shortens in range direction. Also the slant range position of the target is changed in range direction. We note that the double-reflection line moved closer to the time $t_{3}$ as the slant range difference between $t_{2}$ and $t_{3}$ is decreased.

In order to show the interesting replacement effect of these two critical times, we generated the same target with the same height, azimuth size and shape except the slope of the roof but shortened the ground range size of the target to $50 \mathrm{~m}$ 


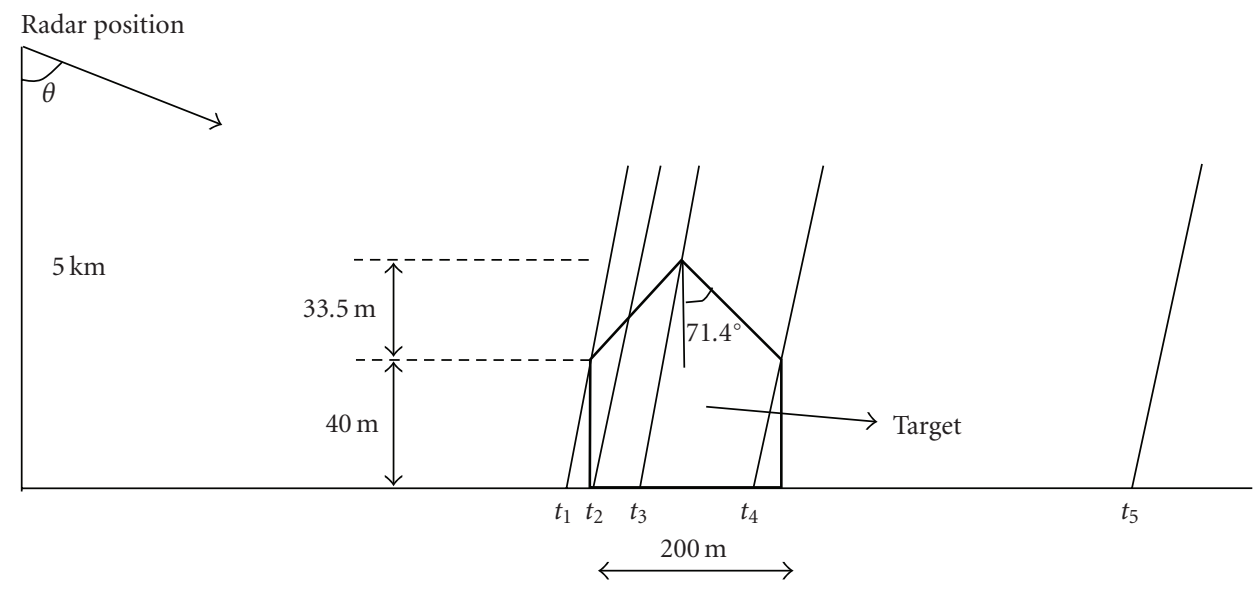

FIgURE 5: Geometry of the radar acquisition.

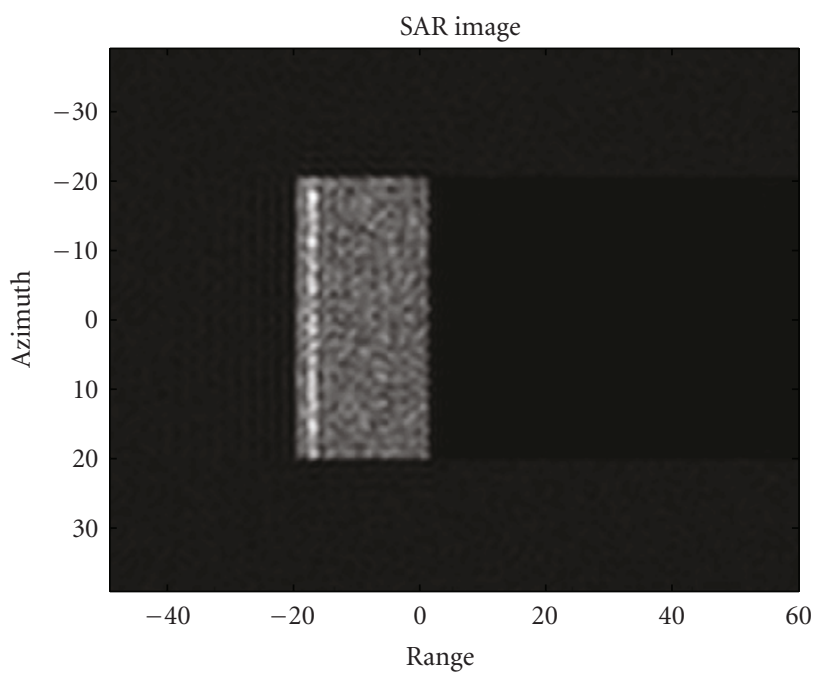

FIGURE 6: Simulated SAR image with aspect angle 75.50.

and generated the SAR raw data with a $10 \mathrm{~km}$ range. The simulated SAR image of this scenario is shown in Figure 7. In this figure, the double reflection line lays on the down slope part of the roof. The backscattering that is generated from the roof is also observed to be replicated weakly due to the sidelobe of backscattering from the up slope part of the target being higher than the shadow.

In order to represent some qualitative results about the target orientation dependency of proposed method, we generated a new target shape in Figure 9. In Figure 9(a), the elevation model of the terrain and the parallelepiped target is shown. The height of each target is $30 \mathrm{~m}$ and rotated in 30 degrees. This target shape is intentionally selected in order to observe both the shadow effects and single and double scattering dependency on target orientation. In Figure 9(b), the absolute value of the computed reflectivity map for the single scattering case is shown. As the roofs and the terrain have the same surface normal, the scattering from both resembles each other. The shadow effects are also observed.

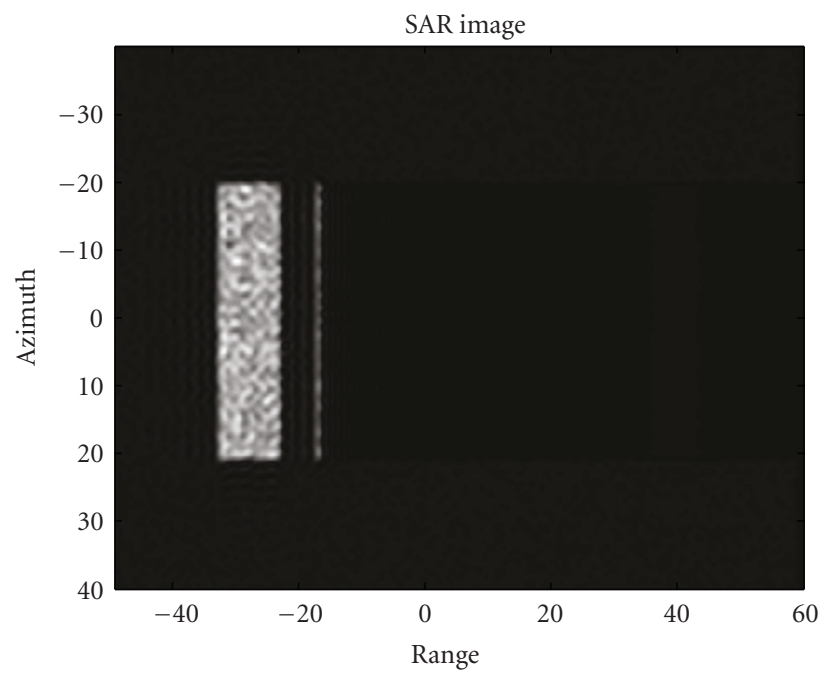

FIgURE 7: Simulated SAR image with aspect angle 63.40.

In Figure 9(c), the absolute value of the reflectivity map of the double scattering effect is shown. It is observed that the vertical surfaces of the targets that is perpendicular to the radar ground range direction, in y direction, generates a more concentrated backscattering, a line, while the rotated ones are more diffused as expected. This diffusion effect cannot be observed in [14]. In Figure 9(d), the processed SAR image is shown, which is a smoothed version of the reflectivity map. As the processed impulse response of the radar is a $2 \mathrm{D}$ sinc function and the SAR image can be analytically defined as a convolution of this $2 \mathrm{D}$ sinc function and the reflectivity map, the smoothening effect is an expected observation. The geometrical features of the SAR images, layover, shadow, and the speckle effects can also be observed in Figure 9(d).

4.2. Time Domain Modularity. In order to show some of the potential modularity advantages of the proposed simulator, in this section, we present some simulation experiments 


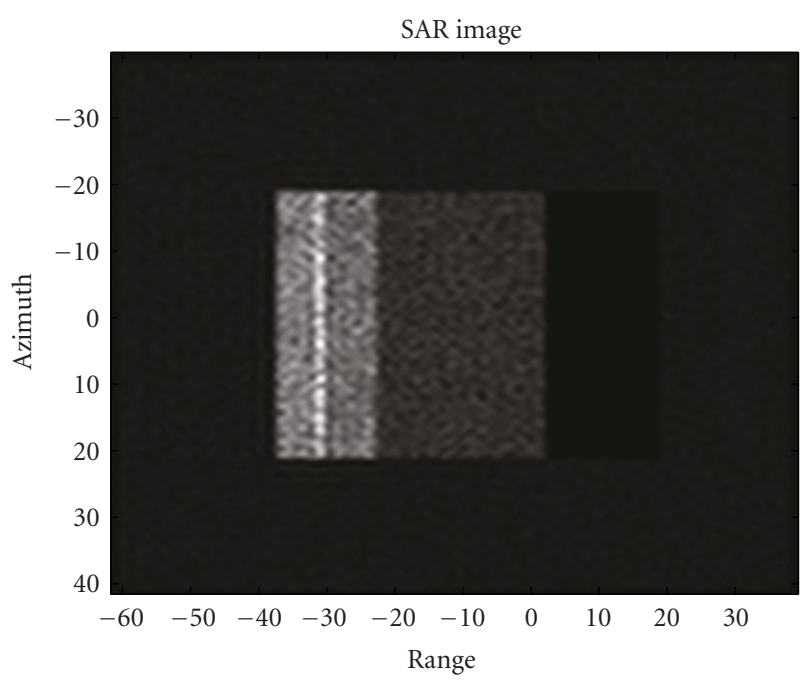

FIGURE 8: Simulated SAR image with aspect angle 51.30.

in the aspect of SAR signal processing. A time domain SAR raw data simulator is known to be time consuming although we proposed a novel procedure in order to increase the efficiency. One shall have the advantage of modularity of such a simulation procedure in order to tolerate the inefficiency. Modularity in terms of SAR raw data generation means handling of different geometrical scenarios and also scattering mechanisms. In this section, in order to validate a time domain simulator, we simply expand our simulator to cover two different geometrical scenarios: the angular deviations of the platform and the moving target case.

Once the 2D matrix of $g\left(x^{\prime}-x, r^{\prime}-r, r\right)$ is generated with a high sampling rate, the rest is to select the right values according to the scenario.

Let us define the range to the target as

$$
r(\eta)=\sqrt{\left(r_{0}+v_{r} \eta\right)^{2}+\left(x-x^{\prime}+v_{a} \eta\right)^{2}}
$$

where $v_{r}$ and $v_{a}$ are used to notate the target movement in range and azimuth direction. If we select the values of the upsampled values of $g\left(x^{\prime}-x, r^{\prime}-r, r\right)$ according to (16) the target will have a movement. We generated the geometry given in Figure 5, with the target moving and obtained the simulated SAR image. The range velocity of the target is $0.5 \mathrm{~m} / \mathrm{s}$, while the azimuth velocity is $10 \mathrm{~m} / \mathrm{sn}$ and the range to the target is $10 \mathrm{~km}$. When we compare the resultant image in Figure 10 with the one in Figure 8, we observe that because of the range velocity an azimuth shift occurs, while because of the azimuth velocity the target image smears. These effects are also expected results. In this aspect this simulator can be utilized to evaluate the performance of moving target detection and imaging algorithms.

For a high radiometric accuracy, the SAR antenna must angularly stabilize itself in order to illuminate the scene uniformly. However especially for the airborne case, this condition cannot be fulfilled exactly. In order to quantify the antenna stabilization performance of a SAR system our proposed work can be modified as follows. According to (10), the upsampled 2D signal is generated ignoring the antenna pattern term, notated as $w^{2}$. For each azimuth rows, the antenna angular deviation terms are generated and multiplied with (10). We emphasize that, this approach already handles the fixed squinted geometry. We generated a sinusoidal deviation of yaw and pitch angles of the antenna with $0.5^{0}$ amplitude and $0.4 \mathrm{~Hz}$ frequency. The simulated SAR image is shown in Figure 11. If we compare this image with the one in Figure 8, we can evaluate the radiometric errors.

One may also define the range to the target considering the platform trajectory deviations in order to simulate a SAR raw data set for evaluation of motion compensation algorithms. Another potential expansion of the simulator can be done by modifying just the wave form of the transmitted signal in order to quantify the performance of different waveforms. We have to emphasize that the main structure of the simulator in order to handle these different scenarios is not changing; therefore the proposed simulator succeed in high flexibility.

4.3. Comparison. In this section we compare our simulator with some of the currently proposed simulators in terms of both raw data computation and reflectivity pattern estimation.

Currently, there are no compromises on the exact metrics of a SAR raw data simulator evaluation. In [17], some criteria for the comparison are proposed. However, these criteria cannot assure that the simulated data represent the real SAR imaging case, either. There are many other concerns that affect a real SAR image like the atmospheric conditions, radar noise figure, polarimetry, and terrain and target interactions. Also for the signal processing view, the motion and angular deviations of a platform, the squinted geometry and the differential range deviation are the other metrics. In order to define the position of our proposed simulator in the literature the comparison table in [17] is extended to cover our simulator, the simulator proposed in [14], and also the additional two criteria: the angular deviation of the antenna and the moving targets cases and presented in Table 3.

As it is shown in Table 3, our proposed simulator and [14] are computing the exact raw data. Margarit et al. published a series of SAR raw data simulators regarding the polarimetry [21], interferometry, simulation of extended sea scenes [22], and analysis of urban areas [23]. In these papers, the raw data of small-sized vessels or urban areas are also computed however, the same procedure cannot be applied in the case of large scenes, as is the case for us, because of the range dependent range cell migration and azimuth chirp rate. That is why we determined the GRECOSAR's capability of SAR raw data simulation as only partially considered. Also this work considers the moving target case however neglects the fixed background. In terms of ground moving target detection and imaging, clutter rejection is an important procedure, and this clutter is the backscattering of the fixed scene. In our work, the raw data is simulated regarding both the fixed scene and the moving target. Another novelty of our simulator is the capability of handling the antenna angular deviations. 


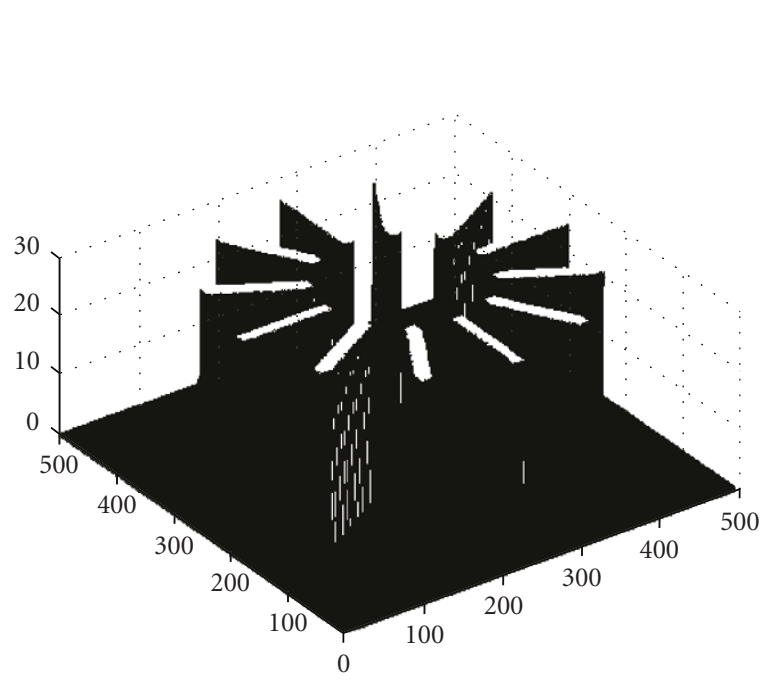

(a)

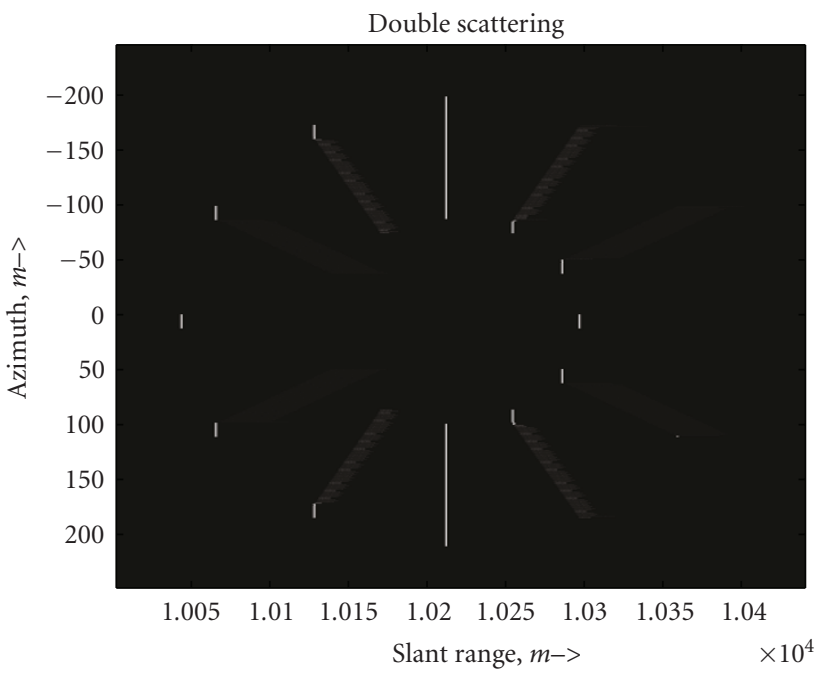

(c)

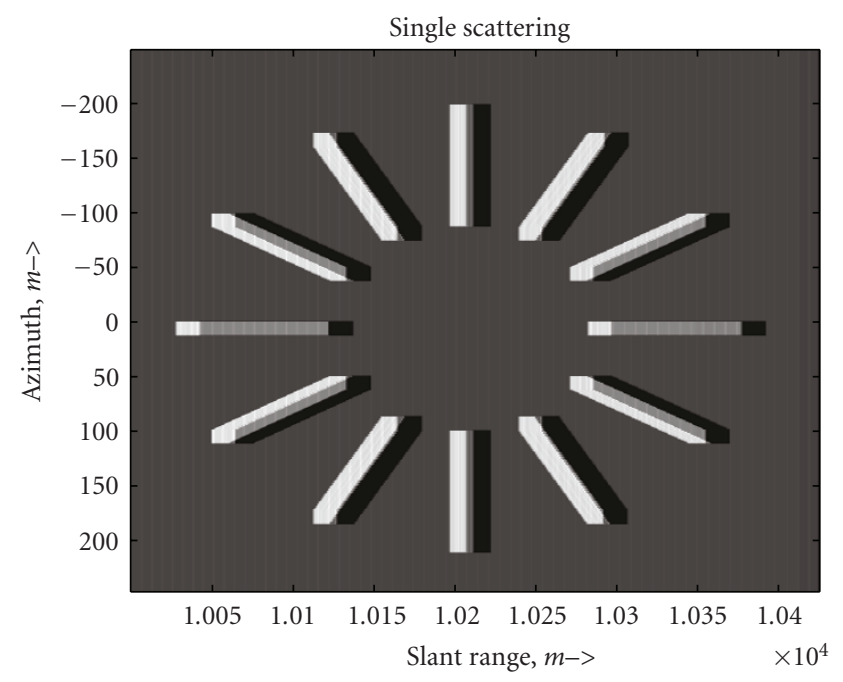

(b)

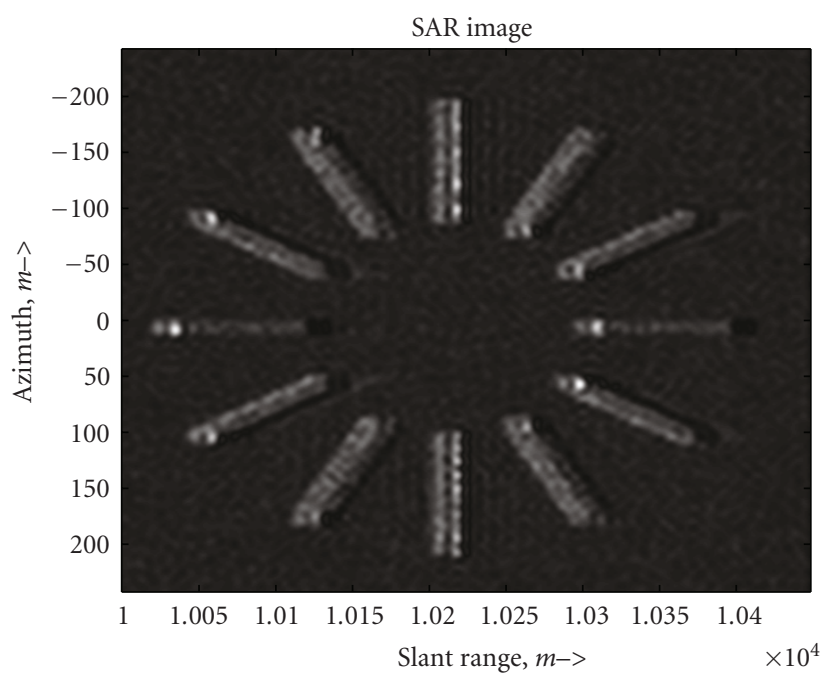

(d)

Figure 9: (a) The input data, (b) the reflectivity map, (c) the double scattering contribution map, and (d) the final SAR image.

TABLE 3: Features considered by SAR simulators: (+) considered, (o) only partially considered, and (-) not considered [12].

\begin{tabular}{|c|c|c|c|c|c|}
\hline Feature & SE-RAY-EM, [18] & GRECOSAR $[19,20]$ & SARViz, [20] & SARAS with [14] & The proposed simulator \\
\hline Layover & + & + & + & + & + \\
\hline Shadows & + & + & + & + & + \\
\hline Double-bounce & + & + & + & + & + \\
\hline Multi-bounce & + & + & - & o & - \\
\hline Materials & + & + & o & o & - \\
\hline Speckle & o & - & o & + & o \\
\hline Real-time & - & - & + & - & - \\
\hline Antenna Deviation & - & - & - & - & + \\
\hline Moving Target Case & - & o & - & - & + \\
\hline Raw Data Simulation & - & o & - & + & + \\
\hline
\end{tabular}




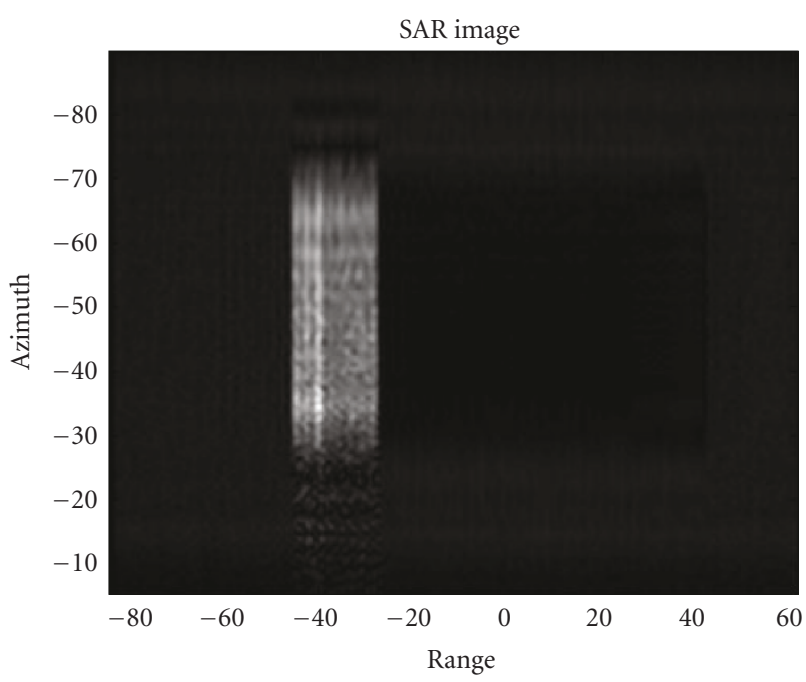

Figure 10: Moving target case.

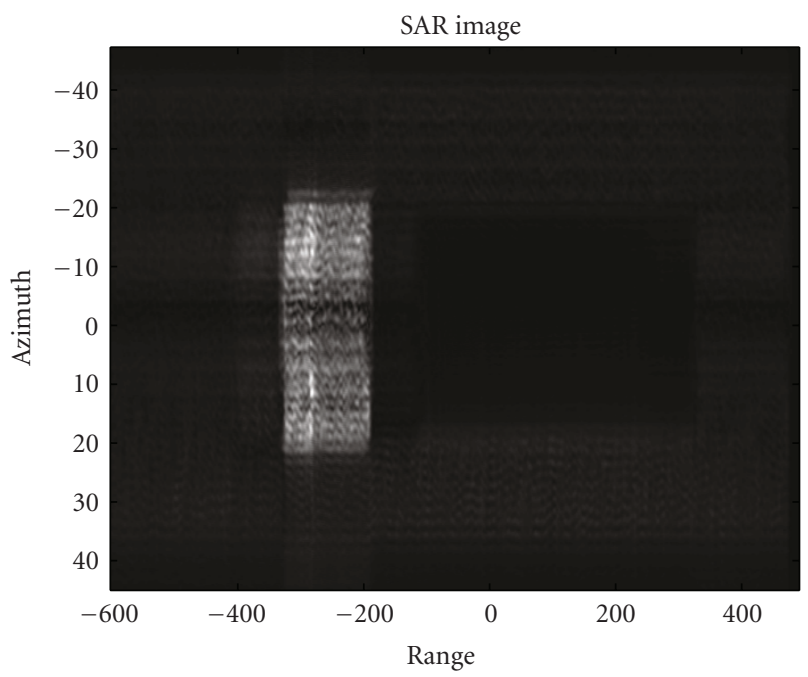

FIgURE 11: Deviating antenna case.

We have to note that only SARViz is a real-time simulator. In terms of reflectivity pattern estimation efficiency, [14] proposes a procedure that the computational time increases dramatically with the number of targets. Our simulator computes the backscattering faster: for a scene of $500 \times$ 500 facets in less than one second. Also in contrast with [14] our simulator is invariant of the number of targets. We have to mention that [14] utilizes an efficient SAR raw data simulation method that is based on frequency domain computation. Unfortunately time domain simulation cannot be implemented such efficiently although we proposed a novel method. For instance the total time to generate the exact SAR raw data in time domain was more than 15 hours for the configuration in Table 2. With the same computer, our proposed method can handle the raw data simulation procedure by less than 4 hours. So the computational efficiency can be increased with this method. Also if the resolution, pulse repetition frequency, or the beamwidth is decreased, the efficiency increases dramatically.

The major weakness of our simulator is neglecting the material properties and the multi-(more than double) bounce. The speckle effect is known to be modeled more accurately in the literature, whereas we only defined an implicit summation of the random phase distributions which is also a reasonable approach [7]. The backscattering is multiplied with a Rayleigh distributed random variable, and the speckle effect is obtained by summation of each facet contribution during SAR raw data computation.

The major contributions of this SAR raw data simulation procedure are as follows. Firstly, we proposed a reasonable criterion to assign the number of facets of the vertical features for double scattering phenomena and taken into consideration the orientation of each facet. Secondly, the simulator computes the reflectivity map very fast although it takes into account the double scattering case. There are other methods to model the backscattering concerning the materials, speckle effects, and triple scattering cases in detail. Here, however, our main purpose is to propose a geometrically accurate and computationally efficient reflectivity map generation and omitting these effects contributed significantly to increase the computational efficiency. From the raw data computation point of view, we proposed a new time domain method that consumes the duration of the procedure in an amount that is related with the resolution, pulse repetition frequency, and beamwidth of the antenna. We proposed a method for the verification of our raw SAR data simulation method. Besides, in many papers, the modularity advantage of time domain simulation was mentioned, however we represented some experiments showing the potentialities of time domain simulation.

\section{Conclusion}

A SAR stripmap mode raw data simulation is proposed. The main goal of this work is to propose a computationally efficient, accurate, and modular SAR raw data simulator that takes into account both the single and double scattering characteristics of an extended scene and the target. The numerical results show that GO-based reflectivity pattern estimation provides a sufficiently accurate computation of the SAR raw data in time-critical applications. We proposed a modular approach by giving the possibility to generate challenging scenarios like moving target and antenna angular deviation cases. In addition, we proposed a new time domain raw data simulation procedure that increases considerably the computational efficiency. For the future works, the method will be expanded to the more realistic airborne cases, like taking into account the positional instabilities of the aircraft.

\section{References}

[1] G. Franceschetti, M. Migliaccio, and D. Riccio, "SAR simulation: an overview," in Proceedings of the International Geoscience and Remote Sensing Symposium (IGARSS '95), pp. 2283-2285, July 1995. 
[2] F. Xu and Y.-Q. Jin, "Imaging simulation of polarimetric SAR for a comprehensive terrain scene using the mapping and projection algorithm," IEEE Transactions on Geoscience and Remote Sensing, vol. 44, no. 11, pp. 3219-3234, 2006.

[3] M. Gelautz, H. Frick, J. Raggam, J. Burgstaller, and F. Leberl, "SAR image simulation and analysis of alpine terrain," ISPRS Journal of Photogrammetry and Remote Sensing, vol. 53, no. 1, pp. 17-38, 1998.

[4] P. W. Vachon, R. K. Raney, and W. J. Emery, "A simulation for spaceborne SAR imagery of a distributed, moving scene," IEEE Transactions on Geoscience and Remote Sensing, vol. 27, no. 1, pp. 67-78, 1989.

[5] Y. Sheng and D. E. Alsdorf, "Automated georeferencing and orthorectification of amazon basin-wide SAR mosaics using SRTM DEM data," IEEE Transactions on Geoscience and Remote Sensing, vol. 43, no. 8, pp. 1929-1940, 2005.

[6] A. Mori and F. De Vita, "A time-domain raw signal simulator for interferometric SAR," IEEE Transactions on Geoscience and Remote Sensing, vol. 42, no. 9, pp. 1811-1817, 2004.

[7] G. Franceschetti, M. Migliaccio, D. Riccio, and G. Schirinzi, "SARAS: a synthetic aperture radar (SAR) raw signal simulator," IEEE Transactions on Geoscience and Remote Sensing, vol. 30, no. 1, pp. 110-123, 1992.

[8] M. Vandewal, R. Speck, and H. Süß, "Efficient SAR raw data generation including low squint angles and platform instabilities," IEEE Geoscience and Remote Sensing Letters, vol. 5, no. 1, pp. 26-30, 2008.

[9] X. Qiu, D. Hu, and L. Zhou, "A bistatic SAR raw data simulator based on inverse $\omega$-k algorithm," IEEE Transactions on Geoscience and Remote Sensing, vol. 48, no. 3, pp. 15401547, 2009.

[10] H. Ling, R. Chou, and S. Lee, "Shooting and bouncing rays: calculating the RCS of an arbitrarily shaped cavity," IEEE Transactions on Antennas and Propagation, vol. 37, no. 2, pp. 194-205, 1989.

[11] G. Franceschetti, A. Iodice, and D. Riccio, "A canonical problem in electromagnetic backscattering from buildings," IEEE Transactions on Geoscience and Remote Sensing, vol. 40, no. 8, pp. 1787-1801, 2002.

[12] F. T. Ulaby and M. C. Dobson, Handbook of Radar Scattering Statistics for Terrain, Artech House, Norwood, Mass, USA, 1989.

[13] G. Franceschetti, A. Iodice, S. Perna, and D. Riccio, "Efficient simulation of airborne SAR raw data of extended scenes," IEEE Transactions on Geoscience and Remote Sensing, vol. 44, no. 10, pp. 2851-2860, 2006.

[14] G. Franceschetti, A. Iodice, D. Riccio, and G. Ruello, "SAR raw signal simulation for urban structures," IEEE Transactions on Geoscience and Remote Sensing, vol. 41, no. 9, pp. 1986-1995, 2003.

[15] W. G. Carrara, R. S. Goodman, and R. M. Majewski, Spotlight Synthetic Aperture Radar: Signal Processing Algorithms, Artech House, Norwood, Mass, USA, 1995.

[16] I. G. Cumming and F. H. Wong, Digital Processing of Synthetic Aperture Radar Data, Artech House, Norwood, Mass, USA, 2005.

[17] H. Hammer, T. Balz, E. Cadario, U. Soergel, U. Thonnessen, and U. Stilla, "Comparison of SAR simulation concepts for the analysis of high-resolution SAR data," in Proceedings of the 7th European Conference on SAR, Friedrichshafen, Germany, 2008.
[18] H. J. Mametsa, F. Rouas, A. Berges, and J. Latger, "Imaging radar simulation in realistic environment using shooting and bouncing rays technique," in SAR Image Analisys, Modeling, and Techniques IV, vol. 4543 of Proceedings of SPIE, pp. 34-40, Toulouse, France, September 2002.

[19] G. Margarit, J. J. Mallorqui, J. M. Rius, and J. Sanz-Marcos, "On the usage of GRECOSAR, an orbital polarimetric SAR simulator of complex targets, to vessel classification studies," IEEE Transactions on Geoscience and Remote Sensing, vol. 44, no. 12, pp. 3517-3525, 2006.

[20] T. Balz and U. Stilla, "Hybrid GPU-based single- and doublebounce SAR simulation," IEEE Transactions on Geoscience and Remote Sensing, vol. 47, no. 10, pp. 3519-3529, 2009.

[21] G. Margarit, J. J. Mallorquí, and X. Fàbregas, "Single-pass polarimetrie SAR interferometry for vessel classification," IEEE Transactions on Geoscience and Remote Sensing, vol. 45, no. 11, pp. 3494-3502, 2007.

[22] G. Margarit, J. J. Mallorqui, J. Fortuny-Guasch, and C. LopezMartinez, "Exploitation of ship scattering in polarimetric SAR for an improved classification under high clutter conditions," IEEE Transactions on Geoscience and Remote Sensing, vol. 47, no. 4, pp. 1224-1235, 2009.

[23] G. Margarit, J. J. Mallorquí, and L. Pipia, "Polarimetric characterization and temporal stability analysis of urban target scattering," IEEE Transactions on Geoscience and Remote Sensing, vol. 48, no. 4, pp. 2038-2048, 2010. 